\title{
Repetibilidade de características do fruto de aceroleira(1)
}

\author{
Ricardo Lopes(2), Claudio Horst Bruckner ${ }^{(3)}$, Cosme Damião Cruz ${ }^{(4)}$, \\ Maria Teresa Gomes Lopes ${ }^{(2)}$ e Gilberto Bernardo de Freitas ${ }^{(3)}$
}

\begin{abstract}
Resumo - Este trabalho teve como objetivo estimar os coeficientes de repetibilidade da altura média de fruto $(\mathrm{AF})$, diâmetro médio de fruto $(\mathrm{DF})$, peso médio de fruto $(\mathrm{PF})$, relação peso da polpa/peso do fruto (RPF), acidez titulável (AT), sólidos solúveis totais (SST) e vitamina $\mathrm{C}$ (VitC), e determinar o número de medidas que devem ser feitas para uma predição acurada do valor real dos indivíduos. Foram utilizados os métodos análise de variância, componentes principais e análise estrutural. As estimativas do coeficiente de repetibilidade das características AF, DF, PF, VitC e AT por ambos os métodos utilizados, demonstraram que coeficientes de determinação $\left(\mathrm{R}^{2}\right)$ acima de $90 \%$ são obtidos a partir de duas medições. Quanto à característica SST, são necessárias, em média, oito medições para alcançar um $\mathrm{R}^{2}$ acima de $90 \%$, e, quanto à característica $\mathrm{RPF}$, no mínimo 26 medições para obter um $\mathrm{R}^{2}$ superior a $90 \%$ o que demonstra não ser viável o aumento do número de medições para alcançar níveis de precisão superiores, e que é necessário buscar um método de determinação da característica que seja mais estável e preciso.
\end{abstract}

Termos para indexação: Malpighia emarginata, propriedades físico-químicas, seleção, genética quantitativa.

\section{Repeatability of fruits traits of acerola tree}

\begin{abstract}
The objective of this paper was to estimate the repeatability coefficient (RC) of the fruit traits such as height (FH), diameter (FD), weight (FW), C vitamin (VitC), total soluble solids (TSS), pulp weight/fruit weight relation (PFR) and the titrable acidity (TA), while the number of measures that should be performed for an efficient selection of the genotypes under evaluation were determined. Analysis were made using the ANOVA, principal components and structural analysis methodology. The estimates of the $\mathrm{RC}$ for $\mathrm{FH}, \mathrm{FD}, \mathrm{FW}, \mathrm{VitC}$ and TA demonstrated high regularity at the superiority of the individuals from one cycle to another, so the evaluation of two cycles was enough to predict the individuals real value with $\mathrm{R}^{2}$ above $90 \%$. To TSS, in average, eight evaluation cycles were necessary to reach a $\mathrm{R}^{2}$ above $90 \%$. PFR showed to be highly irregular, so the evaluation of at least 26 cycles turned to be necessary in order to reach a $\mathrm{R}^{2}$ above $90 \%$. Such an irregularity was attributed to the method used in analyzing the characteristic, then the search for a more accurate and stable method was necessary
\end{abstract}

Index terms: Malpighia emarginata, chemicophysical properties, selection, quantitative genetics.

(1) Aceito para publicação em 23 de junho de 2000. Extraído da dissertação de mestrado apresentada pelo primeiro autor à Universidade Federal de Viçosa (UFV), Viçosa, MG

(2)ESALQ, Dep. de Genética, Av. Pádua Dias, 11, CEP 13418-900 Piracicaba, SP. E-mail: rlopes@carpa.ciagri.usp.br, mtglopes@carpa.ciagri.usp.br

(3)UFV, Dep. de Fitotecnia, CEP 36571-000 Viçosa, MG E-mail: bruckner@mail.ufv.br, bernardo@mail.ufv.br

(4)UFV, Dep. de Biologia Geral. E-mail: cdcruz@mail.ufv.br

\section{Introdução}

Durante o processo de seleção de plantas com vistas ao lançamento de novas cultivares ou à escolha de genitores para recombinação, é importante que se tenha certeza da superioridade genética dos indivíduos. Para tanto, são realizadas, muitas vezes, medições repetidas no mesmo indivíduo. O conhecimento 
do coeficiente de repetibilidade das características de interesse permite avaliar o dispêndio de tempo e mão-de-obra necessários para que a seleção de indivíduos geneticamente superiores seja feita com a acurácia desejada pelo pesquisador. Valores altos da estimativa do coeficiente de repetibilidade do caráter avaliado indicam que é possível predizer o valor real dos indivíduos com um número relativamente pequeno de medições (Cornacchia et al., 1995); isto indica que pouco ganho em acurácia haverá com o aumento do número de medidas repetidas (Falconer, 1987). No entanto, quando a repetibilidade é baixa, grande número de repetições será necessário para que se alcance um valor de determinação satisfatório. O conhecimento do coeficiente de repetibilidade permite, portanto, que a fase de avaliação seja executada com eficiência mas com dispêndio mínimo de tempo e mão-de-obra.

Estimativas de repetibilidade utilizando repetições no tempo têm sido obtidas em culturas perenes, como em seringueira em relação ao caráter produção de borracha (Vasconcellos et al., 1985); em Pinus, quanto às características altura total da planta, diâmetro à altura do peito e volume (Cornacchia et al., 1995), e, em alfafa, quanto às características porcentagem de matéria seca, altura da planta, florescimento, tolerância às doenças e produção de matéria seca (Pereira et al., 1998).

O objetivo deste trabalho foi estimar o coeficiente de repetibilidade de características físicas e químicas de frutos de aceroleira, importantes no processo de seleção, e determinar o número de medidas que devem ser feitas para uma predição acurada do valor real dos indivíduos.

\section{Material e Métodos}

O trabalho foi desenvolvido em delineamento inteiramente casualizado, e consistiu na avaliação de 16 indivíduos com aproximadamente 3,5 anos de idade, nos quais as medições fenotípicas foram repetidas em três ciclos de produção: novembro de 1998, janeiro de 1999, e março de 1999.

Em cada indivíduo foram amostrados 20 frutos do ciclo de produção, e as características avaliadas foram: diâmetro do fruto (DF), medido com paquímetro e expresso em milímetros; altura do fruto (AF), medido com paquímetro e expresso em milímetros; peso médio dos fru- tos (PF), medido com balança digital e expresso em gramas; relação peso da polpa/peso do fruto (RPF), e o peso da polpa foi obtido a partir da diferença do peso total dos frutos e das sementes, as quais, foram extraídas por despolpamento manual, e seu peso foi medido com balança digital; sólidos solúveis totais (SST), determinados por refratometria e expressos em gramas por 100 gramas de polpa; vitamina $\mathrm{C}$ ( VitC), medida por titulação, segundo normas do Instituto Adolfo Lutz (1985) e expressa em miligramas por 100 gramas de polpa; e, enfim, a acidez titulável (AT), medida por titulação, de acordo com as Normas Analíticas do Instituto Adolfo Lutz (1985), e expressa em gramas de ácido málico por 100 gramas de polpa. As análises foram realizadas no Laboratório de $\mathrm{Me}-$ lhoramento de Hortaliças do Departamento de Fitotecnia, da Universidade Federal de Viçosa

As estimativas dos coeficientes de repetibilidade foram obtidas pelos métodos: análise de variância (ANOVA); componentes principais $(\mathrm{CP})$, com base na matriz de correlações e de covariâncias; e análise estrutural (AE), com base na matriz de correlações

Utilizou-se o modelo estatístico com dois fatores de variação (ciclo de produção e indivíduos)

$\mathrm{Y}_{\mathrm{ij}}=\mu+\mathrm{g}_{\mathrm{i}}+\mathrm{a}_{\mathrm{j}}+\varepsilon_{\mathrm{ij}}$,

sendo:

$Y_{\mathrm{ij}}$ : observação referente ao i-ésimo indivíduo na j-ésima medição (ciclo de produção);

$\mu$ : média geral

$\mathrm{g}_{\mathrm{i}}$ : efeito aleatório do i-ésimo indivíduo sob a influência do ambiente permanente ( $\mathrm{i}=1,2, \ldots, 16$ indivíduos);

$a_{j}$ : efeito da j-ésima medição $(j=1,2$ e 3$)$ e,

$\varepsilon_{\mathrm{ij}}$ : erro experimental associado à observação $Y_{\mathrm{ij}}$.

$\mathrm{O}$ esquema da análise de variância é apresentado na Tabela 1

O coeficiente de repetibilidade é dado por:

$r=\frac{\operatorname{COV}\left(Y_{i j}, Y_{i j}\right)}{\sqrt{\hat{V}\left(Y_{i j}\right) \hat{V}\left(Y_{i j}\right)}}=\frac{\hat{\sigma}_{g}^{2}}{\hat{\sigma}_{Y}^{2}}=\frac{\hat{\sigma}_{g}^{2}}{\hat{\sigma}^{2}+\hat{\sigma}_{g}^{2}}$.

O coeficiente de repetibilidade pode ser mais eficientemente estimado por meio da técnica de componentes principais. Numa situação em que o fator periodicidade ocorre, este não pode ser isolado na análise de variância Com isto, este componente é incluído no erro experimental $\left(\sigma^{2}\right)$, elevando seu valor e levando a estimativas não verdadeiras deste componente, e, nesta situação, a repetibilidade é subestimada (Abeywardena, 1972; Kendall, 1975; Vasconcellos et al., 1985).

As estimativas dos coeficientes de repetibilidade foram obtidas pelo método dos componentes principais, com base tanto na matriz de correlações como na matriz de covariâncias entre cada par de medições avaliadas nos diferentes genótipos 
A matriz de correlação paramétrica entre os genótipos em cada par de medições é dada por:

$$
\mathrm{R}=\left[\begin{array}{cccc}
1 & \rho & \ldots & \rho \\
\rho & 1 & \ldots & \rho \\
\ldots & \ldots & \ldots & \ldots \\
\rho & \rho & . . & 1
\end{array}\right]_{\eta},
$$

cujo autovalor $\lambda_{1}$ é dado por $1+(\eta-1) \rho$. Neste estudo considerou-se a matriz $\hat{\mathrm{R}}$ e utilizou-se o estimador do coeficiente de repetibilidade proposto por Rutledge (1974), que é dado por:

$\hat{\mathrm{r}}=\frac{\hat{\lambda}_{1}-1}{\eta-1}$,

onde:

$\eta$ : número de períodos avaliados, $\mathrm{e}$

$\hat{\lambda}_{1}$ : autovalor obtido da matriz $\hat{\mathrm{R}}$ associado ao autovetor cujos elementos têm o mesmo sinal e magnitudes semelhantes.

O coeficiente de repetibilidade foi também estimado considerando a matriz paramétrica de variâncias e covariâncias fenotípicas

$$
\Gamma=\sigma_{Y}^{2}\left[\begin{array}{cccc}
1 & \rho & \ldots & \rho \\
\rho & 1 & \ldots & \rho \\
\ldots & \ldots & \ldots & \ldots \\
\rho & \rho & . . & 1
\end{array}\right]_{\eta},
$$

cujo autovalor $\hat{\lambda}_{1}$ é dado por

$$
\sigma_{\mathrm{Y}}^{2}[1+(\eta-1) \rho] \text {. }
$$

Considerou-se a matriz $\hat{\Gamma}$ e obteve-se o estimador da repetibilidade por meio de:

$$
\hat{\mathrm{r}}=\frac{\hat{\lambda}_{1}-\hat{\sigma}_{\mathrm{Y}}^{2}}{\hat{\sigma}_{\mathrm{Y}}^{2}(\eta-1)} \text {, em que } \hat{\sigma}_{\mathrm{Y}}^{2}=\hat{\sigma}^{2}+\hat{\sigma}_{\mathrm{g}}^{2} \text {, }
$$

sendo $\hat{\lambda}_{1}$ o autovalor obtido de $\hat{\Gamma}$ associado ao autovetor cujos elementos têm o mesmo sinal e magnitudes semelhante.

Foram obtidas as estimativas de repetibilidade pelo método da análise estrutural, com base na matriz de corre-

Tabela 1. Esquema da análise de variância no modelo com dois fatores de variação.

\begin{tabular}{lccc}
\hline Fonte de variação & GL & QM & $\mathrm{E}(\mathrm{QM})$ \\
\hline Indivíduo & $\mathrm{p}-1$ & $\mathrm{QMG}$ & $\sigma^{2}+3 \sigma_{\mathrm{g}}^{2}$ \\
Ciclo de produção & $\mathrm{a}-1$ & $\mathrm{QME}$ & - \\
Resíduo & $(\mathrm{p}-1)(\mathrm{a}-1)$ & $\mathrm{QMR}$ & $\sigma^{2}$ \\
\hline
\end{tabular}

lações entre os genótipos em cada par de avaliação proposto por Mansour et al. (1981).

Este método considera $\mathrm{R}$ a matriz paramétrica de correlações entre genótipos em cada par de avaliação, e $\hat{R}$, seu estimador. Neste caso, o estimador do coeficiente de repetibilidade é dado por:

$\hat{\mathrm{r}}=\frac{\mathrm{a}^{\prime} \hat{\mathrm{R}} \mathrm{a}-1}{\eta-1}$,

sendo $a^{\prime}=[1 / \sqrt{\eta} \ldots 1 / \sqrt{\eta}]$ o autovetor com elementos paramétricos, associados ao maior autovalor obtido de $\mathrm{R}$.

De acordo com Cruz \& Regazzi (1997), este estimador é a média aritmética das correlações fenotípicas entre genótipos, considerando cada par de medições, ou seja: $\mathrm{r}=\frac{2}{\eta(\eta-1)} \sum_{\mathrm{j}<\mathrm{j}} \sum_{\mathrm{j}} \mathrm{r}_{\mathrm{jj}}$.

Uma vez estimado o coeficiente de repetibilidade (r), a estimativa do número de medições $\left(\eta_{0}\right)$ necessárias para se predizer o valor real dos indivíduos com o valor de determinação genotípica $\left(\mathrm{R}^{2}\right)$ desejado é obtida pela expressão:

$\eta_{0}=\frac{R^{2}(1-\hat{r})}{\left(1-R^{2}\right) \hat{r}}$.

$\mathrm{O}$ coeficiente de determinação genotípica $\left(\mathrm{R}^{2}\right)$, que representa a porcentagem de certeza da predição do valor real dos indivíduos selecionados com base em $\eta$ medições é obtido pela expressão:

$$
\mathrm{R}^{2}=\frac{\eta \mathrm{r}}{1+\mathrm{r}(\eta-1)} \text {. }
$$

As estimativas foram obtidas através do procedimento repetibilidade do programa GENES (Cruz, 1997).

\section{Resultados e Discussão}

Os resultados da análise de variância utilizandose o modelo com dois fatores de variação (indivíduos e ciclo de produção), provenientes das medidas realizadas em três ciclos de produção em 16 indivíduos para as sete características em estudo são apresentados na Tabela 2. Com exceção da relação peso da polpa/peso do fruto (RPF), cujo valor de $\mathrm{F}$ não foi significativo, indicando, assim, inexistência de variação entre os indivíduos, quanto às demais características, os valores de $\mathrm{F}$ apresentaram-se significativos a $1 \%$, evidenciando, portanto, a existência de variabilidade destas características entre os indivíduos.

As estimativas do coeficiente de repetibilidade obtidas, com relação às sete características por meio 
dos quatro métodos utilizados, encontram-se nas Tabelas 3 a 6 . As estimativas do coeficiente de repetibilidade obtidas pelo método da análise de variância são sempre menores ou iguais às estimativas obtidas pelos demais métodos, e que pelo método dos componentes principais (baseado na matriz de covariâncias) estas estimativas foram sempre maiores ou iguais às obtidas pelos demais métodos. No entanto, as diferenças observadas entre as estimativas obtidas pelos diferentes métodos são pequenas, e na maioria dos casos não alteram o número de medições em um mesmo nível de precisão.

Trabalhos nos quais foram obtidas e comparadas estimativas de repetibilidade de características de plantas perenes pelo método da ANOVA e métodos multivariados (análise estrutural e componentes principais), como seringueira (Vasconcellos et al., 1985) e Pinus (Cornacchia et al., 1995), mostraram que as estimativas obtidas pelo método da ANOVA foram sempre inferiores às obtidas pela análise multivariada.

Segundo Cruz \& Regazzi (1997), o método da análise estrutural proposto por Mansour et al. (1981) apresenta apenas diferenças conceituais em relação ao método dos componentes principais, e portanto, as estimativas obtidas pelos respectivos métodos tendem a ser próximas. Quando os valores das estimativas do coeficiente de repetibilidade são menores, a diferença entre os resultados obtidos pelos diferentes métodos aumenta, como ocorre com as caracte- rísticas RPF e SST, fato também verificado por Vasconcellos et al. (1985).

O método da ANOVA não permite isolar o fator periodicidade, que, quando ocorre, fica incluído no erro experimental $\left(\sigma^{2}\right)$, elevando seu valor, e então, a repetibilidade é subestimada (Vasconcellos et al., 1985). Neste caso, o coeficiente de repetibilidade é mais eficientemente estimado pelo método dos componentes principais, que leva em consideração o comportamento cíclico do caráter (Kendall, 1975).

Com relação às características $\mathrm{AF}, \mathrm{DF}, \mathrm{PF}$, VitC e AT, as estimativas obtidas pelos quatro métodos utilizados demonstraram acurácia das medições realizadas, alta regularidade da superioridade dos indivíduos de um ciclo para outro, e que a expressão das características tem bom controle genético. Coeficientes de determinação superiores a $90 \%$ são obtidos para estas características, a partir de duas medições, e este coeficiente é superior a $95 \%$ para PF. Para predição de valores reais dos indivíduos com coeficiente de determinação acima de $95 \%$, são necessárias, em média, quatro repetições.

A repetibilidade representa o valor máximo que a herdabilidade no sentido amplo pode atingir (Cruz \& Regazzi, 1997). A diferença entre a repetibilidade e a herdabilidade se deve ao fato de que a variância genotípica utilizada para estimar a repetibilidade não é somente de origem genética, uma vez que o componente de variância do ambiente permanente entre

Tabela 2. Análise de variância das variáveis diâmetro médio de fruto (DF), altura média de fruto (AF), peso médio de fruto $(\mathrm{PF})$, relação peso da polpa/peso do fruto (RPF), sólidos solúveis totais (SST), vitamina C (VitC) e acidez titulável (AT) medidas em três ciclos de produção em 16 indivíduos, e estimativas dos componentes de variância entre genótipos e variância ambiental.

\begin{tabular}{lcccccccc}
\hline Fonte de variação & Graus de & \multicolumn{7}{c}{ QM das características } \\
\cline { 2 - 8 } & liberdade & $\mathrm{DF}$ & $\mathrm{AF}$ & $\mathrm{PF}$ & $\mathrm{RPF}$ & $\mathrm{SST}$ & $\mathrm{VitC}$ & $\mathrm{AT}$ \\
\hline Ciclo de produção & 2 & 5,0889 & 6,0077 & 4,6837 & 0,00040 & 4,0527 & 164545,89 & 0,4415 \\
Indivíduo & 15 & $43,4184^{* *}$ & $18,7815^{* *}$ & $27,2990^{* *}$ & $0,00030^{\text {ns }}$ & $1,3552^{* *}$ & $595107,85^{* *}$ & $0,4092^{* *}$ \\
Resíduo & 30 & 1,8180 & 1,1939 & 0,7623 & 0,00027 & 0,3187 & 33488,25 & 0,0290 \\
Média & & 22,59 & 19,10 & 6,10 & 0,90 & 6,47 & 1468,13 & 1,34 \\
$\mathrm{CV}(\%)$ & & 5,96 & 5,71 & 14,29 & 1,84 & 8,72 & 12,46 & 12,64 \\
$\hat{\sigma}_{\mathrm{g}}^{2}$ & & 13,86 & 5,86 & 8,84 & $9,2 \times 10^{-6}$ & 0,34 & 187206,53 & 0,12 \\
$\hat{\sigma}^{2}$ & & 1,81 & 1,19 & 0,76 & $2,7 \times 10^{-4}$ & 0,31 & 33488,25 & 0,02 \\
\hline
\end{tabular}

ns Não-significativo. ${ }^{* *}$ Significativo a $1 \%$ de probabilidade 
indivíduos permanece confundido com esta. Assim, a repetibilidade aproxima-se da herdabilidade à medida que a variância proporcionada pelos efeitos permanentes do ambiente é minimizada. Se a variância genotípica estimada fosse puramente de natureza genética, os coeficientes de repetibilidade estimados corresponderiam à herdabilidade das características.

A característica SST apresentou estimativa de repetibilidade variando de 0,52 pelo método da ANOVA a 0,59 pelo método dos CP (baseado na matriz de covariâncias), o que evidencia baixa regularidade na repetição do caráter de um ciclo para

Tabela 3. Estimativas dos coeficientes de repetibilidade $(\hat{\mathrm{r}})$, coeficientes de determinação $\left(\mathrm{R}^{2}\right)$ e do número de medições calculados $\left(\eta_{0}\right)$, utilizando o método da análise de variância para as características altura média de fruto $(\mathrm{AF})$, diâmetro médio de fruto $(\mathrm{DF})$, peso médio de fruto $(\mathrm{PF})$, relação peso da polpa/peso do fruto (RPF), sólidos solúveis totais ( $\mathrm{SST})$, vitamina $\mathrm{C}$ (VitC) e acidez titulável (AT), medidas em três ciclos de produção em 16 genótipos de aceroleira.

\begin{tabular}{|c|c|c|c|c|}
\hline \multirow[t]{2}{*}{ Característica } & \multicolumn{2}{|c|}{$\begin{array}{l}\text { Valor obtido a } \\
\text { partir de três } \\
\text { medições }\end{array}$} & \multicolumn{2}{|c|}{$\begin{array}{c}\text { Número de medições } \\
\text { necessárias para } \\
\text { diferentes coeficientes } \\
\text { de determinação }\left(\mathrm{R}^{2}\right) \\
\end{array}$} \\
\hline & $\hat{\mathrm{r}}$ & $\mathrm{R}^{2}(\%)$ & $\mathrm{R}^{2}$ & $\eta_{0}^{(1)}$ \\
\hline \multirow[t]{3}{*}{$\mathrm{AF}$} & 0,83 & 93,64 & 0,90 & $2(1,84)$ \\
\hline & & & 0,95 & $4(3,89)$ \\
\hline & & & 0,99 & $21(20,27)$ \\
\hline \multirow[t]{3}{*}{ DF } & 0,88 & 95,81 & 0,90 & $2(1,22)$ \\
\hline & & & 0,95 & $3(2,59)$ \\
\hline & & & 0,99 & $14(13,5)$ \\
\hline \multirow[t]{3}{*}{ PF } & 0,92 & 97,20 & 0,90 & $1(0,78)$ \\
\hline & & & 0,95 & $2(1,65)$ \\
\hline & & & 0,99 & $9(8,60)$ \\
\hline \multirow[t]{3}{*}{ RPF } & 0,03 & 9,06 & 0,90 & $291(291)$ \\
\hline & & & 0,95 & $615(614,33)$ \\
\hline & & & 0,99 & 3201 (3201) \\
\hline \multirow[t]{3}{*}{ SST } & 0,52 & 76,48 & 0,90 & $9(8,3)$ \\
\hline & & & 0,95 & $18(17,53)$ \\
\hline & & & 0,99 & $92(91,38)$ \\
\hline \multirow[t]{3}{*}{ VitC } & 0,84 & 94,37 & 0,90 & $2(1,71)$ \\
\hline & & & 0,95 & $4(3,61)$ \\
\hline & & & 0,99 & $19(18,85)$ \\
\hline \multirow[t]{3}{*}{ AT } & 0,81 & 92,90 & 0,90 & $3(2,11)$ \\
\hline & & & 0,95 & $5(4,45)$ \\
\hline & & & 0,99 & $24(23,22)$ \\
\hline
\end{tabular}

(1)Número aproximado (número calculado). outro. Para esta característica, são necessárias, em média, oito repetições para que sejam alcançados coeficientes de determinação superiores a 90\%.

Na seleção para a característica SST, constata-se não ser vantajoso o aumento do número de repetições visando maior nível de certeza na predição do valor real dos indivíduos. Porém, com quatro medições, seria possível obter um coeficiente de determinação acima de $80 \%$.

A estimativa do coeficiente de repetibilidade obtida para relação peso da polpa/peso do fruto foi muito baixa, variando de 0,03 pelo método da ANOVA a

Tabela 4. Estimativas dos coeficientes de repetibilidade $(\hat{\mathrm{r}})$, coeficientes de determinação $\left(\mathrm{R}^{2}\right)$ e do número de medições calculados $\left(\eta_{0}\right)$, utilizando o método dos componentes principais baseado na matriz de correlações para as características altura média de fruto (AF), diâmetro médio de fruto $(\mathrm{DF})$, peso médio de fruto $(\mathrm{PF})$, relação peso da polpa/peso do fruto (RPF), sólidos solúveis totais (SST), vitamina C (VitC) e acidez titulável (AT), medidas em três ciclos de produção em 16 genótipos de aceroleira.

\begin{tabular}{cccccc}
\hline Característica & $\begin{array}{c}\text { Valor obtido a } \\
\text { partir de três } \\
\text { medições }\end{array}$ & & \multicolumn{2}{c}{$\begin{array}{c}\text { Número de medições } \\
\text { necessárias para } \\
\text { diferentes coeficientes } \\
\text { de determinação }\left(\mathrm{R}^{2}\right)\end{array}$} \\
\cline { 2 - 3 } \cline { 5 - 6 } & $\hat{\mathrm{r}}$ & $\mathrm{R}^{2}(\%)$ & & $\mathrm{R}^{2}$ & $\eta_{0}{ }^{(1)}$ \\
\hline AF & 0,84 & 94,37 & & 0,90 & $2(1,71)$ \\
& & & & 0,95 & $4(3,61)$ \\
DF & 0,88 & 96,01 & & 0,90 & $2(1,22)$ \\
& & & 0,95 & $3(2,59)$ \\
& & & 0,99 & $14(13,5)$ \\
PF & 0,94 & 97,94 & 0,90 & $1(0,95)$ \\
& & & 0,95 & $2(1,21)$ \\
RPF & 0,16 & 37,83 & 0,90 & $48(47,25)$ \\
& & & 0,95 & $100(99,75)$ \\
& & & 0,99 & $520(519,75)$ \\
SST & 0,55 & 79,18 & 0,90 & $8(7,36)$ \\
& & & 0,95 & $16(15,54)$ \\
& & & 0,99 & $81(81)$ \\
VitC & 0,85 & 94,73 & 0,90 & $2(1,58)$ \\
& & & 0,95 & $4(3,35)$ \\
& & & 0,99 & $18(17,47)$ \\
AT & 0,84 & 94,04 & 0,90 & $2(1,71)$ \\
& & & 0,95 & $4(3,61)$ \\
& & & 0,99 & $19(18,85)$ \\
\hline
\end{tabular}

(1)Número aproximado (número calculado).

Pesq. agropec. bras., Brasília, v. 36, n. 3, p. 507-513, mar. 2001 
0,26 pelo método dos $\mathrm{CP}$ (baseado na matriz de covariâncias), indicando grande irregularidade da superioridade dos indivíduos de um ciclo para outro. Para alcançar um coeficiente de determinação superior a 90\%, seriam necessárias 291 medições a partir da estimativa obtida pelo método da ANOVA, e 26 medições pela estimativa do coeficiente de repetibilidade obtida pelo método dos $\mathrm{CP}$ (baseado na matriz de covariâncias). Esta alta irregularidade é atribuída em maior parte à ineficiência do método de determinação da característica utilizado, sendo ne- cessário que sejam testados métodos mais eficientes, ou seja, de análise mais precisa, para determinar a relação peso da polpa/peso do fruto. Constata-se, portanto, que é inviável aumentar o número de repetições objetivando alcançar um coeficiente de determinação satisfatório mantendo o mesmo método de determinação da relação peso da polpa/peso do fruto.

A baixa precisão na predição do valor real dos indivíduos das características RPF e SST, mesmo considerando a imprecisão do método de determina-
Tabela 5. Estimativas dos coeficientes de repetibilidade $(\hat{\mathrm{r}})$, coeficientes de determinação $\left(\mathrm{R}^{2}\right)$ e do número de medições calculados $\left(\eta_{0}\right)$, utilizando o método dos componentes principais baseado na matriz de covariâncias para as características altura média de fruto (AF), diâmetro médio de fruto (DF), peso médio de fruto $(\mathrm{PF})$, relação peso da polpa/peso do fruto (RPF), sólidos solúveis totais (SST), vitamina C (VitC) e acidez titulável (AT), medidas em três ciclos de produção em 16 genótipos de aceroleira

\begin{tabular}{|c|c|c|c|c|}
\hline \multirow[t]{2}{*}{ Característica } & \multicolumn{2}{|c|}{$\begin{array}{l}\text { Valor obtido a } \\
\text { partir de três } \\
\text { medições }\end{array}$} & \multicolumn{2}{|c|}{$\begin{array}{l}\text { Número de medições } \\
\text { necessárias para } \\
\text { diferentes coeficientes } \\
\text { de determinação }\left(\mathrm{R}^{2}\right)\end{array}$} \\
\hline & $\hat{\mathrm{r}}$ & $\mathrm{R}^{2}(\%)$ & $\mathrm{R}^{2}$ & $\eta_{0}^{(1)}$ \\
\hline \multirow[t]{3}{*}{$\mathrm{AF}$} & 0,85 & 94,79 & 0,90 & $2(1,58)$ \\
\hline & & & 0,95 & $4(3,35)$ \\
\hline & & & 0,99 & $18(17,47)$ \\
\hline \multirow[t]{3}{*}{ DF } & 0,89 & 96,11 & 0,90 & $2(1,11)$ \\
\hline & & & 0,95 & $3(2,34)$ \\
\hline & & & 0,99 & $13(12,23)$ \\
\hline \multirow[t]{3}{*}{$\mathrm{PF}$} & 0,94 & 98,16 & 0,90 & $1(0,64)$ \\
\hline & & & 0,95 & $2(1,21)$ \\
\hline & & & 0,99 & $7(6,31)$ \\
\hline \multirow[t]{3}{*}{ RPF } & 0,26 & 51,47 & 0,90 & $26(25,61)$ \\
\hline & & & 0,95 & $54(54,07)$ \\
\hline & & & 0,99 & $282(281,76)$ \\
\hline \multirow[t]{3}{*}{ SST } & 0,59 & 81,65 & 0,90 & $7(6,25)$ \\
\hline & & & 0,95 & $14(13,20)$ \\
\hline & & & 0,99 & $69(68,79)$ \\
\hline \multirow[t]{3}{*}{ VitC } & 0,85 & 94,75 & 0,90 & $2(1,58)$ \\
\hline & & & 0,95 & $4(3,35)$ \\
\hline & & & 0,99 & $18(17,47)$ \\
\hline \multirow[t]{3}{*}{$\mathrm{AT}$} & 0,85 & 94,59 & 0,90 & $2(1,58)$ \\
\hline & & & 0,95 & $4(3,35)$ \\
\hline & & & 0,99 & $18(17,47)$ \\
\hline
\end{tabular}

Pesq. agropec. bras., Brasília, v. 36, n. 3, p. 507-513, mar. 2001
Tabela 6. Estimativas dos coeficientes de repetibilidade $(\hat{\mathrm{r}})$, coefícientes de determinação $\left(\mathrm{R}^{2}\right)$ e do número de medições calculados $\left(\eta_{0}\right)$, utilizando o método da análise estrutural baseado na matriz de correlações para as características altura média de fruto $(\mathrm{AF})$, diâmetro médio de fruto (DF), peso médio de fruto (PF), relação peso da polpa/peso do fruto (RPF), sólidos solúveis totais (SST), vitamina $\mathrm{C}$ ( $\mathrm{VitC}$ ) e acidez titulável (AT), medidas em três ciclos de produção em 16 genótipos de aceroleira.

\begin{tabular}{|c|c|c|c|c|}
\hline \multirow[t]{2}{*}{ Característica } & \multicolumn{2}{|c|}{$\begin{array}{l}\text { Valor obtido a } \\
\text { partir de três } \\
\text { medições }\end{array}$} & \multicolumn{2}{|c|}{$\begin{array}{c}\text { Número de medições } \\
\text { necessárias para } \\
\text { diferentes coeficientes } \\
\text { de determinação }\left(\mathrm{R}^{2}\right)\end{array}$} \\
\hline & $\hat{\mathrm{r}}$ & $\mathrm{R}^{2}(\%)$ & $\mathrm{R}^{2}$ & $\eta_{0}{ }^{(1)}$ \\
\hline \multirow[t]{3}{*}{$\mathrm{AF}$} & 0,84 & 94,36 & 0,90 & $2(1,71)$ \\
\hline & & & 0,95 & $4(3,61)$ \\
\hline & & & 0,99 & $19(18,85)$ \\
\hline \multirow[t]{3}{*}{ DF } & 0,88 & 96,00 & 0,90 & $2(1,22)$ \\
\hline & & & 0,95 & $3(2,59)$ \\
\hline & & & 0,99 & $14(13,5)$ \\
\hline \multirow[t]{3}{*}{$\mathrm{PF}$} & 0,94 & 97,94 & 0,90 & $1(0,57)$ \\
\hline & & & 0,95 & $2(1,21)$ \\
\hline & & & 0,99 & $7(6,31)$ \\
\hline \multirow[t]{3}{*}{ RPF } & 0,06 & 18,00 & 0,90 & $141(141)$ \\
\hline & & & 0,95 & $298(297,66)$ \\
\hline & & & 0,99 & $1551(1551)$ \\
\hline \multirow[t]{3}{*}{ SST } & 0,54 & 78,34 & 0,90 & $8(7,66)$ \\
\hline & & & 0,95 & $17(16,18)$ \\
\hline & & & 0,99 & $85(84,33)$ \\
\hline \multirow[t]{3}{*}{ VitC } & 0,85 & 94,73 & 0,90 & $2(1,58)$ \\
\hline & & & 0,95 & $4(3,35)$ \\
\hline & & & 0,99 & $18(17,47)$ \\
\hline \multirow[t]{3}{*}{ AT } & 0,84 & 94,03 & 0,90 & $2(1,71)$ \\
\hline & & & 0,95 & $4(3,61)$ \\
\hline & & & 0,99 & $19(18,85)$ \\
\hline
\end{tabular}

(1)Número aproximado (número calculado) 
ção do RPF, demonstra que essas características são as mais influenciadas pelo ambiente, e que a seleção indireta via características de melhor controle genético seria uma boa estratégia.

\section{Conclusões}

1. As estimativas dos coeficientes de repetibilidade das características altura média de fruto, diâmetro médio de fruto, peso médio de fruto, vitamina $\mathrm{C}$ e acidez titulável demonstram alta regularidade na superioridade dos indivíduos de um ciclo para outro, e que dois ciclos de avaliação são suficientes para predizer o valor real dos indivíduos com nível de certeza acima de $90 \%$.

2. Não são constatadas grandes diferenças entre as estimativas dos coeficientes de repetibilidade obtidos pelo método da ANOVA e pelos métodos de análise multivariada.

3. O baixo valor do coeficiente de repetibilidade da característica relação peso da polpa/peso do fruto revela alta irregularidade de um ciclo para outro, deste modo, o aumento no número de medições pouco acrescenta em precisão.

4. Apesar da característica sólidos solúveis totais ter considerável influência ambiental, três medições permitem alcançar coeficientes de determinação próximos a $80 \%$

\section{Referências}

ABEYWARDENA, V. An application of component analysis in genetics. Journal of Genetics, Sadashivanagar v. 61, p. $27-51,1972$.

CORNACCHIA, G.; CRUZ, C. D.; LOBO, P. R.; PIRES, I. E. Estimativas do coeficiente de repetibilidade para ca- racterísticas fenotípicas de procedências de Pinus tecunumanii (Schw.) Eguiluz, Perry e Pinus caribaea var. hondurensis Barret, Golfari. Revista Árvore, Viçosa, v. 19 , n. 3 , p. $333-345,1995$

CRUZ, C. D. Programa GENeS: aplicativo computacional em genética e estatística. Viçosa : UFV, 1997. $442 \mathrm{p}$

CRUZ, C. D.; REGAZZI, A. J. Modelos biométricos aplicados ao melhoramento genético. 2. ed. Viçosa : UFV, 1997. $390 \mathrm{p}$

FALCONER, D. S. Introdução a genética quantitativa Viçosa : UFV, 1987. 279 p

INSTITUTO ADOLFO LUTZ (São Paulo, SP). Normas analíticas do Instituto Adolfo Lutz. 3. ed. São Paulo, 1985. $533 \mathrm{p}$.

KENDALL, M. G. Multivariate analysis. New York : MacMillan, 1975. 210 p.

MANSOUR, H.; NORDHEIM, E. V.; RUTLEDGE, J. J. Estimations of repeatability. Theoretical and Applied Genetics, Berlin, v. 60, p. 151-156, 1981

PEREIRA, A. V.; FERREIRA, R. P.; CRUZ, C. D.; FREITAS, V. P.; OLIVEIRA, T. A. Comportamento de alfafa $\mathrm{cv}$. Crioula de diferentes origens e estimativas dos coeficientes de repetibilidade para caracteres forrageiros. Revista Brasileira de Zootecnia, Viçosa, v. 27, n. 4, p. $686-690,1998$

RUTLEDGE, J. J. A scaling which removes bias of Abeywardena's estimator of repeatability. Journal Genetics, Sadashivanagar, v. 61, p. 247-250, 1974.

VASCONCELLOS, M. E. C.; GONÇALVES, P. S.; PAIVA, J. R.; VALOIS, A. C. C. Métodos de estimação do coeficiente de repetibilidade no melhoramento da seringueira. Pesquisa Agropecuária Brasileira, Brasília, v. 20, n. 4 , p. 433-437, abr. 1985 